\title{
Combination therapy with methadone and duloxetine for cancer- related pain: a retrospective study
}

\author{
Zachary A. Curry ${ }^{1}$, Malisa C. Dang ${ }^{1}$, Adam P. Sima ${ }^{2}$, Sura Abdullaziz ${ }^{1}$, Egidio G. Del Fabbro ${ }^{1}$ \\ ${ }^{1}$ Department of Internal Medicine, Division of Hematology, Oncology, and Palliative Care, Virginia Commonwealth University, Richmond, VA, \\ USA; ${ }^{2}$ Department of Biostatistics, Virginia Commonwealth University, Richmond, VA, USA \\ Contributions: (I) Conception and design: ZA Curry, MC Dang, EG Del Fabbro; (II) Administrative support: MC Dang; (III) Provision of study \\ materials or patients: None; (IV) Collection and assembly of data: ZA Curry, MC Dang, S Abdullaziz, EG Del Fabbro; (V) Data analysis and \\ interpretation: ZA Curry, MC Dang, AP Sima, EG Del Fabbro; (VI) Manuscript writing: All authors; (VII) Final approval of manuscript: All authors. \\ Correspondence to: Zachary A. Curry, MD, PhD. 1101 E Marshall Street Box 980230 Richmond, VA 23298, USA. Email: curryza@vcu.edu.
}

\begin{abstract}
Background A comprehensive approach to pain management often requires multimodal therapy and a combination of medications. Oncology patients may be prescribed methadone and duloxetine as single agents or in combination for cancer-related pain, particularly neuropathic pain. Duloxetine is also prescribed for depression or anxiety in patients with cancer.

Methods: A retrospective chart review on patients with cancer-related pain prescribed duloxetine and methadone combination therapy at the Virginia Commonwealth University supportive care clinic (SCC) between 2012 and 2019. Edmonton Symptom Assessment System (ESAS) scores reported by patients on monotherapy were compared to scores after they started combination therapy. Of 131 patients identified on combination therapy, 43 met study criteria (2 with incomplete ESAS scores).

Results: ESAS total and subscores after combination therapy were lower than on monotherapy. Combination therapy decreased total, pain, and emotion subscores by $5.6\left(\mathrm{SD}=17.3, \mathrm{~d}_{\mathrm{z}}=-0.32, \mathrm{P}=0.046\right), 0.9$ $\left(\mathrm{SD}=3.0, \mathrm{~d}_{\mathrm{z}}=-0.30, \mathrm{P}=0.052\right)$, and $1.8\left(\mathrm{SD}=5.1, \mathrm{~d}_{\mathrm{z}}=-0.36, \mathrm{P}=0.023\right)$, respectively. On combination therapy, $28 \%$ of patients reported at least a two-point reduction in pain scores. All study participants reported cancer pain with neuropathic components; most had mixed pain syndromes comprising nociceptive and neuropathic components. Adherence rates were high as $81 \%$ of patients with follow-up appointments continued therapy.

Conclusions: These results suggest the combination of duloxetine and methadone reduces cancer-related pain and emotional symptom burden compared to either medication as a single agent.
\end{abstract}

Keywords: Cancer pain; supportive care; duloxetine; methadone

Submitted Jul 31, 2020. Accepted for publication Nov 25, 2020.

doi: 10.21037/apm-20-1455

View this article at: http://dx.doi.org/10.21037/apm-20-1455

\section{Introduction}

Opioids remain the mainstay treatment for cancer pain despite concerns about the potential for misuse and failure to achieve adequate pain control in almost half of oncology patients (1-4). Pain management requires a comprehensive approach, often with multimodal therapy and a combination of medications (1). Both methadone and the antidepressant duloxetine are prescribed for cancer related pain, particularly neuropathic pain or mixed pain syndromes having a combination of nociceptive and neuropathic pain (5-7). Methadone, a potent $\mu$ opioid receptor agonist and NMDA receptor antagonist has unique properties including low cost, high oral bioavailability, and a long halflife allowing for dosing two to three times per day $(1,8,9)$. A limited systematic review of methadone suggests similar effectiveness to morphine for moderate or severe cancer pain with lower financial cost $(5,10,11)$. 
Duloxetine, an antidepressant, is a dual serotonin and norepinephrine reuptake inhibitor that significantly decreases pain scores and improves quality of life in chemotherapy induced peripheral neuropathy $(7,12)$. The addition of duloxetine to opioid-pregabalin combination therapy significantly reduced cancer related neuropathic pain, but did not improve quality of life scores or emotional function (13).

Given the efficacy of methadone and duloxetine for treatment-resistant cancer pain, we hypothesized that the combination of duloxetine and methadone (combination therapy) improves measures of pain and other symptoms compared to monotherapy with either agent alone. In this study, we conducted a retrospective chart review examining measures of pain and symptom burden in cancer patients before and after starting combination therapy. The objective of this study was to determine if the combination of methadone and duloxetine improve symptom burden, particularly neuropathic pain, in cancer patients. We present the following article in accordance with the STROBE reporting checklist (available at http://dx.doi.org/10.21037/ apm-20-1455).

\section{Methods}

This study was conducted in accordance with the Declaration of Helsinki (as revised in 2013). The study was approved by the institutional review board of Virginia Commonwealth University (protocol No. HM20017599) and individual consent for this retrospective analysis was waived.

\section{Study design}

A retrospective chart review on ambulatory oncology patients with cancer pain treated in the supportive care clinic (SCC) at Virginia Commonwealth University, a National Cancer Institute (NCI) designated cancer center. The Cancer Informatics Core at Massey Cancer Center conducted a search of the Massey Data Analysis System (MDAS) for patients prescribed both duloxetine and methadone in 2012-2019. The Institutional Review Board (IRB) at Virginia Commonwealth University approved this study.

Data were collected and managed using Research Electronic Data Capture (REDCap), a secure webbased application. Inclusion criteria for patients included: (I) $\geq 18$ years of age; (II) a current or previous cancer diagnosis; (III) were receiving methadone or duloxetine prescribed by the SCC; (IV) had visited the SCC at least twice with cancer-related pain. Patients were excluded if visit notes indicated lack of combination therapy, noncompliance with therapy, pain unrelated to cancer diagnosis, or visits falling outside of the proposed study design.

The Edmonton Symptom Assessment System (ESAS) was chosen as it is well-validated, rapid assessment test administered in the SCC (14). ESAS scores including total, physical (pain, tiredness, nausea, drowsiness, appetite, shortness of breath), and emotional (depression and anxiety) were collected from visit notes before and after initiation of combination therapy (Figure 1) (15). At the initial visit, patients were required to have a pre-existing prescription for duloxetine or methadone (monotherapy) and then received a new prescription for the second agent. The follow-up visit (combination therapy) was the latest patient encounter within 2-8 weeks of starting combination therapy. This timeline was chosen to ensure an adequate trial of medication to include multiple half-lives of methadone, allow for duloxetine or methadone dose adjustment, identify treatment benefit, and measure adherence to therapy. To estimate patient acceptability of combination therapy, a third and final visit was collected within $2-8$ weeks from the follow-up visit. Other factors collected and potentially associated with treatment outcomes included demographic data, methadone and duloxetine total daily dosages, type of cancer, cancer classification as non-advanced versus advanced (locally recurrent or metastatic), and diagnosed or reported pain type (nociceptive, neuropathic, or mixed). Cancer pain syndromes were classified by either recorded clinician diagnosis or assigned based on documented patientreported features with neuropathic pain assigned to patients with numbness, tingling, burning, or radicular features (16). For patients starting methadone, the rotation strategy (no, partial, or complete) describing change from preexisting opioid therapy was reported. Patients either started methadone as a new drug (no rotation), underwent partial rotation (switched long-acting opioid to methadone), or complete rotation (switched all opioids to methadone) (17).

\section{Statistical analysis}

Patient demographic information was summarized using means and standard deviations or frequencies and percentages separately for each combination therapy group. Insurance information was included as a marker of 


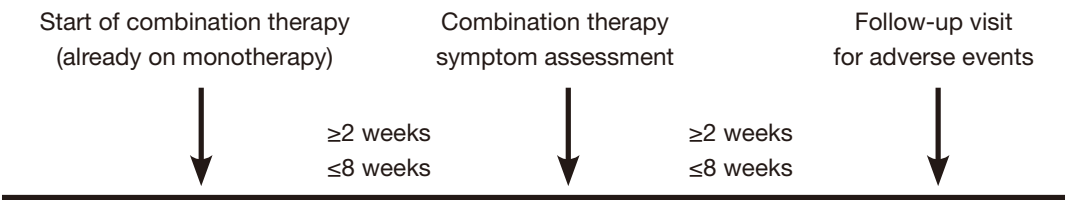

Figure 1 Study design. Edmonton Symptom Assessment System (ESAS) scores were recorded at the start of combination therapy (patient already prescribed duloxetine or methadone), during combinatin therapy (prescribed both duloxetine and methadone), and at a final followup visit. Each subsequent visit was at least two weeks or at most eight weeks after the prior visit.

socioeconomic status. The differences between the ESAS total, subscores, and individual items between combination and monotherapy were assessed using paired t-tests across each initial treatment type. Standardized differences $\left(d_{z}\right)$ were reported for each ESAS score (18). Standardized differences $>0.8$ were interpreted as a high difference, $0.5-$ 0.8 as a medium difference, $0.2-0.5$ as a small difference. The frequency of patients responding to combination therapy, as defined as a 2-point reduction in subscores, were also reported (13). SAS V9.4 was used for statistical analysis and figure generation. Missing data were excluded from analysis.

\section{Results}

Of the 131 patients identified during screening, 43 patients received combination therapy within the study timeline (25 starting methadone, 18 starting duloxetine). One patient from each group lacked baseline drowsiness and total ESAS scores for analysis. Baseline patient characteristics, drug doses, and baseline median ESAS total and subscores for both groups are listed in Table 1. Of those with a third visit 2-8 weeks after combination therapy ( $\mathrm{N}=32), 81 \%$ of patients were still on combination therapy. Almost all patients $(\mathrm{N}=40)$ reported features of both nociceptive and neuropathic cancer pain with three patients (one in the duloxetine-methadone group, two in methadone-duloxetine group) reporting primarily neuropathic pain (one in each group with chemotherapy neuropathy and one with postsurgical pain).

ESAS total and subscores after combination therapy were lower than on monotherapy. Decreases of 5.6 $\left(\mathrm{SD}=17.3, \mathrm{~d}_{\mathrm{z}}=-0.32, \mathrm{P}=0.046\right), 0.9\left(\mathrm{SD}=3.0, \mathrm{~d}_{\mathrm{z}}=-0.30\right.$, $\mathrm{P}=0.052), 2.8\left(\mathrm{SD}=12.3, \mathrm{~d}_{\mathrm{z}}=-0.23, \mathrm{P}=0.150\right)$, and $1.8(\mathrm{SD}$ $\left.=5.1, \mathrm{~d}_{\mathrm{z}}=-0.36, \mathrm{P}=0.023\right)$ were observed in the total, pain, physical, and emotion subscores, respectively (Figure 2A,B). On combination therapy, $28 \%$ and $33 \%$ of all patients responded with at least 2 - and 1-point reductions in pain scores, respectively, with similar results for other subscores (Table 2). All individual items demonstrated decreases from monotherapy with anxiety showing the largest decrease from monotherapy to combination therapy $(1.1, \mathrm{SD}=2.8$, $\mathrm{d}_{\mathrm{z}}=-0.38, \mathrm{P}=0.018$; Figure 2). Difference information for other subscores are reported in Table S1.

For patients on duloxetine starting methadone, 44\% $(\mathrm{N}=11)$ had no rotation, 24\% $(\mathrm{N}=6)$ underwent partial rotation, and $32 \%(\mathrm{~N}=8)$ underwent complete rotation. Patients with no evidence of disease (NED) at the time of treatment included those with breast cancer treated with mastectomy, acute lymphoblastic leukemia treated with allogenic stem cell transplant, chemotherapy-related neuropathy, and other cancers (colon, rectum, anal) after definitive treatment. All patients with NED reported components of nociceptive and neuropathic pain with the exception of two patients with chemotherapy-related neuropathic pain.

\section{Discussion}

This study shows combination therapy with methadone and duloxetine reduced symptom burden in oncology patients already receiving monotherapy with either drug. Combination therapy decreased all measures of symptom burden and produced notable changes in pain, physical, and emotion scores with the greatest decrease in the anxiety subscore. This combination was well-tolerated despite the majority of patients having advanced cancer. All patients reported features of cancer-related neuropathic pain and high levels of pain at baseline, suggesting inadequate management on monotherapy alone.

Although a systematic review of 8 trials reported opioid and antidepressant combination pharmacotherapy reduced neuropathic pain in cancer patients, another meta-analysis recommended clinicians should balance the small likelihood of benefit for cancer pain, including neuropathic pain, against the increased risk of adverse effects of combination 
Table 1 Summary of group characteristics

\begin{tabular}{|c|c|c|}
\hline Variable & Duloxetine + methadone $(n=25)$ & Methadone + duloxetine $(n=18)$ \\
\hline Sex (female) & $14(56 \%)$ & $9(50 \%)$ \\
\hline \multicolumn{3}{|l|}{ Race } \\
\hline White & $15(60 \%)$ & $12(67 \%)$ \\
\hline Other & $0(0 \%)$ & $1(6 \%)$ \\
\hline \multicolumn{3}{|l|}{ Insurance type } \\
\hline Medicare/Medicaid & $14(56 \%)$ & $8(44 \%)$ \\
\hline Commercial/HMO & $6(24 \%)$ & $4(22 \%)$ \\
\hline \multicolumn{3}{|l|}{ Cancer classification } \\
\hline Advanced disease & $23(92 \%)$ & $12(67 \%)$ \\
\hline No evidence of disease & $2(8 \%)$ & $6(33 \%)$ \\
\hline Monotherapy dose (median, mg) & 60 & 33.75 \\
\hline \multicolumn{3}{|c|}{ Combination therapy dose (median, mg) } \\
\hline Duloxetine & 60 & 40 \\
\hline Methadone & 15 & 30 \\
\hline \multicolumn{3}{|c|}{ ESAS scores (combination start, median) } \\
\hline Drowsiness & 3 & 1 \\
\hline Appetite & 5 & 2.5 \\
\hline Wellbeing & 4 & 5 \\
\hline Shortness of breath & 2 & 1 \\
\hline Sleep/rest & 6 & 4 \\
\hline Total ESAS & 38.5 & 39 \\
\hline
\end{tabular}

ESAS, Edmonton Symptom Assessment System; HMO, Health Maintenance Organization.

therapy $(19,20)$. Our study is the first to report the benefits of combining methadone and duloxetine for pain and symptom burden in patients with cancer. A randomized controlled trial of duloxetine added to opioid-pregabalin therapy alleviated refractory neuropathic cancer pain
$(13,21)$, but in contrast to our results the addition of duloxetine to opioid-pregabalin therapy did not decrease overall symptom burden or emotional symptom burden scores (13). Patients also reported worsening of appetiterelated symptoms, which was not significantly altered in our 
A

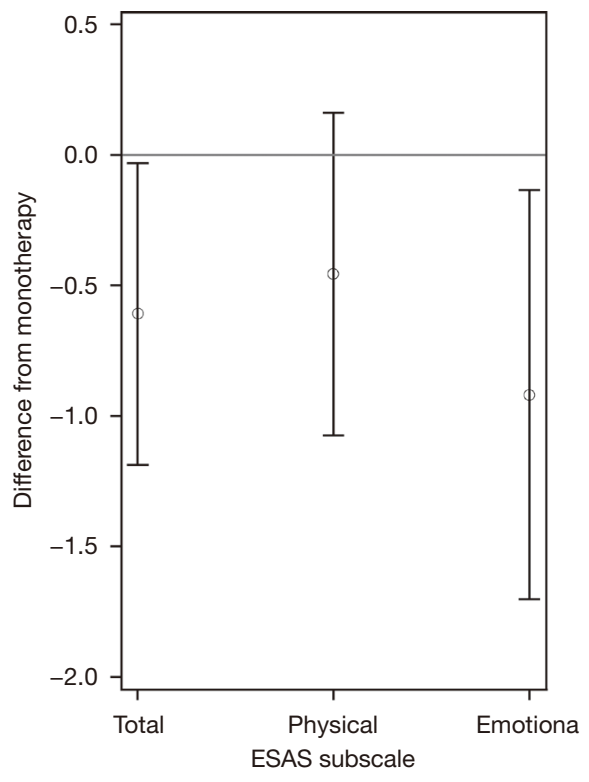

B

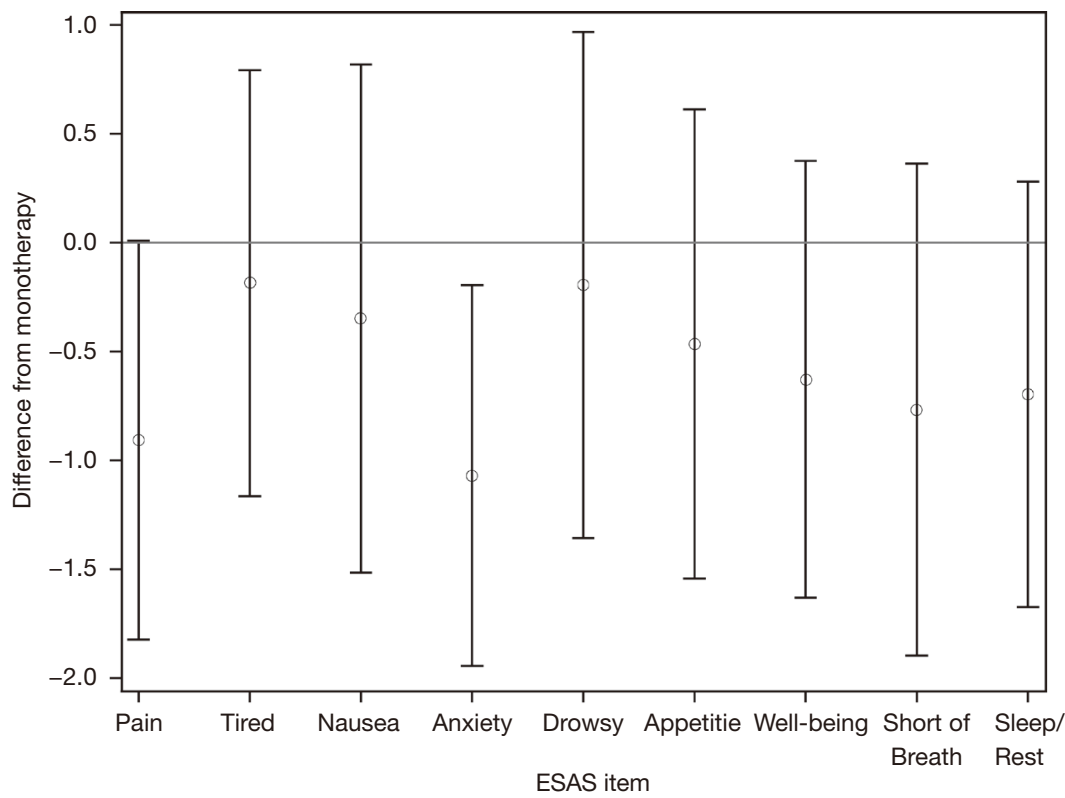

Figure 2 Combination therapy reduces symptom burden in cancer patients. (A) Compared to monotherapy, the combination of methadone and duloxetine reduces Edmonton Symptom Assessment System (ESAS) total [5.6, standard deviation (SD) =17.3], physical (2.8, SD =12.3), and emotion $(1.8, \mathrm{SD}=5.1)$ scores in oncology patients. (B) Pain $(0.9, \mathrm{SD}=3.0)$, anxiety $(1.1, \mathrm{SD}=2.8)$ and other scores were also reduced in oncology patients. Data are reported at mean and $95 \%$ confidence interval. Total, physical, and emotional scores were standardized (std) to have a range of $0-10$.

Table 2 Frequency of patients with a two-point and one-point reduction following combination therapy

\begin{tabular}{lcc}
\hline ESAS measure & $\begin{array}{c}\text { Two-point } \\
\text { responders }\end{array}$ & $\begin{array}{c}\text { One-point } \\
\text { responders }\end{array}$ \\
\hline Pain & $12(28 \%)$ & $14(33 \%)$ \\
Tired & $8(19 \%)$ & $13(30 \%)$ \\
Nausea & $12(28 \%)$ & $13(30 \%)$ \\
Anxious & $10(23 \%)$ & $14(33 \%)$ \\
Drowsy & $7(17 \%)$ & $12(29 \%)$ \\
Appetite & $9(21 \%)$ & $13(30 \%)$ \\
Well-being & $13(30 \%)$ & $17(40 \%)$ \\
Shortness of breath & $10(23 \%)$ & $11(26 \%)$ \\
Sleep/rest & $14(33 \%)$ & $16(37 \%)$ \\
\hline
\end{tabular}

ESAS, Edmonton Symptom Assessment System.

study (13).

Although a Cochrane review finds that no conclusions can be made for the efficacy or safety between methadone and placebo, other opioids, or other treatments for neuropathic pain, the use of low dose methadone as an adjuvant analgesic in patients with cancer pain is being explored (22-25). Emerging evidence suggests methadone is more effective than fentanyl for neuropathic pain in head and neck cancer, and rotation to methadone in oncology patients with mixed pain syndromes (a combination of nociceptive and neuropathic pain) showed superior efficacy compared to other opioids while decreasing the number of analgesic prescriptions (5). Other types of pain also appear to respond to the unique properties of methadone, including intraoperative use of methadone which reduces postoperative pain scores and produces better patient satisfaction scores compared to other opioids (26). While methadone is not typically prescribed for anxiety treatment, improved emotional scores noted in our study may be due to pain relief and improved physical symptom burden or a direct effect on the serotonin system (27).

The high adherence rate for our study is notable as patients would have to tolerate at least four weeks of combination therapy. This is in contrast to a clinical trial using the combination of duloxetine and methadone for HIV-associated polyneuropathy which was limited 
by recruitment and retention with only 8 of 15 patients completing the study (28). Factors cited for study failure included stringent inclusion/exclusion criteria, regulatory challenges related to methadone acquisition, and adverse effects due to methadone use by opioid-naïve patients (28). However, in patients on methadone for opioid use disorder, the addition of desvenlafaxine (a serotonin-norepinephrine reuptake inhibitor similar to duloxetine) for depression was well-tolerated with $86 \%$ adherence to treatment (29).

This study has several limitations including the retrospective nature and the relatively small sample size from a single NCI center. Although all patients were diagnosed with or reported features typical of neuropathic pain, the ability to accurately diagnose neuropathic pain based on retrospective chart review is difficult. Most of the patients had a mixed pain syndrome. The strengths of our study include the use of the ESAS, a validated, reliable tool used for longitudinal monitoring in patients seen by palliative care (14). Additionally, the use of a standardized protocol, adhering to a strict time frame when assessing patients on combination therapy, allowing for several half-lives of methadone, increases the reliability of our findings (1).

Our preliminary observational study supports combination therapy for cancer related pain and is the first to report methadone and duloxetine as an effective combination for reducing pain, anxiety, and overall symptom burden when monotherapy is insufficient. This study also lays the groundwork for future clinical trials in validating the effectiveness of combination therapy and identifying predictors of combination therapy response.

\section{Acknowledgments}

Funding: Support of this research project was provided by Virginia Commonwealth University Massey Cancer Center Informatics Core with funding, in part, from the National Institutes of Health-National Cancer Institute (NIH-NCI) Cancer Center Support Grant P30 CA016059. Dr. Del Fabbro also receives research support from NIH grants R01AG061558 and R01CA225701.

\section{Footnote}

Reporting Checklist: The authors have completed the STROBE reporting checklist. Available at http://dx.doi. org/10.21037/apm-20-1455

Data Sharing Statement: Available at http://dx.doi. org/10.21037/apm-20-1455

Conflicts of Interest: All authors have completed the ICMJE uniform disclosure form (available at http://dx.doi. org/10.21037/apm-20-1455). Dr. EGDF serves as an unpaid editorial board member of Annals of Palliative Medicine from Mar 2019 to Feb 2021. The other authors have no conflicts of interest to declare.

Ethical Statement: The authors are accountable for all aspects of the work in ensuring that questions related to the accuracy or integrity of any part of the work are appropriately investigated and resolved. This study was conducted in accordance with the Declaration of Helsinki (as revised in 2013). The study was approved by the institutional review board of Virginia Commonwealth University (protocol No. HM20017599) and individual consent for this retrospective analysis was waived.

Open Access Statement: This is an Open Access article distributed in accordance with the Creative Commons Attribution-NonCommercial-NoDerivs 4.0 International License (CC BY-NC-ND 4.0), which permits the noncommercial replication and distribution of the article with the strict proviso that no changes or edits are made and the original work is properly cited (including links to both the formal publication through the relevant DOI and the license). See: https://creativecommons.org/licenses/by-nc-nd/4.0/.

\section{References}

1. Portenoy RK. Treatment of cancer pain. Lancet 2011;377:2236-47.

2. Greco MT, Roberto A, Corli O, et al. Quality of cancer pain management: An update of a systematic review of undertreatment of patients with cancer. J Clin Oncol 2014;32:4149-54.

3. Del Fabbro E. Assessment and management of chemical coping in patients with cancer. J Clin Oncol 2014;32:1734-8.

4. Deandrea S, Montanari M, Moja L, et al. Prevalence of undertreatment in cancer pain. A review of published literature. Ann Oncol 2008;19:1985-91.

5. Haumann J, Geurts JW, van Kuijk SMJ, et al. Methadone is superior to fentanyl in treating neuropathic pain in patients with head-and-neck cancer. Eur J Cancer 2016;65:121-9.

6. Rhondali W, Tremellat F, Ledoux M, et al. Methadone Rotation for Cancer Patients with Refractory Pain in a 
Palliative Care Unit: An Observational Study. J Palliat Med 2013;16:1382-7.

7. Smith EM, Pang H, Cirrincione C, et al. Effect of Duloxetine on Pain, Function, and Quality of Life Among Patients With Chemotherapy-Induced Painful Peripheral Neuropathy. JAMA 2013;309:1359-67.

8. Madden K, Mills S, Dibaj S, et al. Methadone as the Initial Long-Acting Opioid in Children with Advanced Cancer. J Palliat Med 2018;21:1317-21.

9. González-Barboteo J, Porta-Sales J, Nabal-Vicuña M, et al. Switching Ratio from Parenteral to Oral Methadone 1:1.2 Is Safer Compared with Ratio 1:2 in Patients with Controlled Cancer Pain: A Multicenter RandomizedControlled Trial (RATIOMTD-010810). J Palliat Med 2021;24:382-90.

10. Sugiyama Y, Sakamoto N, Ohsawa M, et al. A Retrospective Study on the Effectiveness of Switching to Oral Methadone for Relieving Severe Cancer-Related Neuropathic Pain and Limiting Adjuvant Analgesic Use in Japan. J Palliat Med 2016;19:1051-9.

11. Nicholson AB, Watson G, Derry S, et al. Methadone for cancer pain. Cochrane Database Syst Rev 2017;(2):CD003971.

12. Colvin LA. Chemotherapy-induced peripheral neuropathy: where are we now? Pain 2019;160:S1-10.

13. Matsuoka H, Iwase S, Miyaji T, et al. Additive Duloxetine for Cancer-Related Neuropathic Pain Nonresponsive or Intolerant to Opioid-Pregabalin Therapy: A Randomized Controlled Trial (JORTC-PAL08). J Pain Symptom Manage 2019;58:645-53.

14. Hui D, Bruera E. The Edmonton Symptom Assessment System 25 Years Later: Past, Present, and Future Developments. J Pain Symptom Manage 2017;53:630-43.

15. Richardson LA, Jones GW. A review of the reliability and validity of the Edmonton Symptom Assessment System. Curr Oncol 2009; 16:55-64.

16. Yoon SY, Oh J. Neuropathic cancer pain: Prevalence, pathophysiology, and management. Korean J Intern Med 2018;33:1058-69.

17. Reddy A, Yennurajalingam S, Pulivarthi K, et al. Frequency, outcome, and predictors of success within 6 weeks of an opioid rotation among outpatients with cancer receiving strong opioids. Oncologist 2013;18:212-20.

18. Cohen J. Statistical power analysis for the behavioral sciences. 2nd ed. Hillsbale, NJ: Lawrence Erlbaum Associates; 1988.

19. Kane CM, Mulvey MR, Wright S, et al. Opioids combined with antidepressants or antiepileptic drugs for cancer pain: Systematic review and meta-analysis. Palliat Med 2018;32:276-86.

20. Guan J, Tanaka S, Kawakami K. Anticonvulsants or antidepressants in combination pharmacotherapy for treatment of neuropathic pain in cancer patients. Clin J Pain 2016;32:719-25.

21. Matsuoka H, Iwase S, Miyaji T, et al. Predictors of duloxetine response in patients with neuropathic cancer pain: a secondary analysis of a randomized controlled trial-JORTC-PAL08 (DIRECT) study. Support Care Cancer 2020;28:2931-9.

22. Chalker C, O'Neill H, Cranfield F. Efficacy of low-dose and/or adjuvant methadone in palliative medicine. BMJ Support Palliat Care 2019. [Epub ahead of print]. doi: 10.1136/bmjspcare-2018-001695.

23. Fürst P, Lundström S, Klepstad P, et al. Improved Pain Control in Terminally Ill Cancer Patients by Introducing Low-Dose Oral Methadone in Addition to Ongoing Opioid Treatment. J Palliat Med 2018;21:177-81.

24. Courtemanche F, Dao D, Gagné F, et al. Methadone as a Coanalgesic for Palliative Care Cancer Patients. J Palliat Med 2016;19:972-8.

25. McNicol ED, Ferguson M, Schumann R. Methadone for neuropathic pain in adults. Cochrane Database Syst Rev 2017;5:CD012499.

26. Machado FC, Vieira JE, de Orange FA, et al. Intraoperative Methadone Reduces Pain and Opioid Consumption in Acute Postoperative Pain: A Systematic Review and MetaAnalysis. Anesth Analg 2019;129:1723-32.

27. Fogaça MV, Fukumoto K, Franklin T, et al. N-Methyl$\mathrm{D}$-aspartate receptor antagonist $\mathrm{d}$-methadone produces rapid, mTORC1-dependent antidepressant effects. Neuropsychopharmacology 2019;44:2230-8.

28. Harrison T, Miyahara S, Lee A, et al. Experience and challenges presented by a multicenter crossover study of combination analgesic therapy for the treatment of painful HIV-associated polyneuropathies. Pain Med 2013;14:1039-47.

29. El Hage C, Ghabrash MF, Dubreucq S, et al. A pilot, open-label, 8-week study evaluating desvenlafaxine for treatment of major depression in methadonemaintained individuals with opioid use disorder. Int Clin Psychopharmacol 2018;33:268-73.

Cite this article as: Curry ZA, Dang MC, Sima AP, Abdullaziz S, Del Fabbro EG. Combination therapy with methadone and duloxetine for cancer-related pain: a retrospective study. Ann Palliat Med 2021;10(3):2505-2511. doi: 10.21037/apm-20-1455 
Supplementary

Table S1 Change from monotherapy to combination therapy in each of the additional Edmonton Symptom Assessment System subscores

\begin{tabular}{lccc}
\hline ESAS measure & Difference (SD) & $\mathrm{P}$ & $\mathrm{d} z$ \\
\hline Tired & $-0.2(3.2)$ & 0.703 & -0.06 \\
Nausea & $-0.3(3.8)$ & 0.550 & -0.09 \\
Anxious & $-1.1(2.8)$ & 0.018 & -0.38 \\
Drowsy & $-0.2(3.7)$ & 0.736 & -0.05 \\
Appetite & $-0.5(3.5)$ & 0.389 & -0.13 \\
Well-being & $-0.6(3.3)$ & 0.213 & -0.20 \\
Shortness of breath & $-0.8(3.7)$ & 0.178 & -0.21 \\
Sleep/rest & $-0.7(3.2)$ & 0.157 & -0.22 \\
\hline
\end{tabular}

ESAS, Edmonton Symptom Assessment System; SD, standard deviation; $P, P$ value; $d$, standardized difference. 